\title{
Relationship between Mineragraphy Features of Sinter Ore and Its Gray Histogram
}

\author{
Xuewei LV, Chenguang BAI, Guibao QIU, Meilong HU and Shengfu ZHANG \\ College of Materials Science \& Engineering, Chongqing University, Chongqing, 400044, P. R. China. \\ E-mail: Ivxuewei@163.com, bguang@cqu.edu.cn, qiuguibao@163.com, qtml2003@163.com, zhang65109609@sina.com
}

(Received on September 3, 2007; accepted on December 3, 2007)

\begin{abstract}
The intelligent recognizing and processing system make a great convenience for recognition and measurement of sinter ore mineragraphy. Specially, it's crucial for a successful intelligent system to extract the minerals' features accurately and sufficiently. The paper got the relatively position of peak of the gray histogram in theory by using the calculated model of index of reflection and peak-find model of gray histogram; the parameters like $\mu$ and $\theta^{2}$ of familiar minerals were gotten by statistical averaging with Gaussian gray distribution model, the information about the category and content of minerals can also be got by fitting the curve of gray histogram with Gaussian gray distribution model; the feature curves of gray histogram of two minerals were gained by synthesizing two density functions; also the feature parameters such as the number and position of peak and valley of two minerals in different ratios were obtained by differentiating to the distribution functions. The feature parameters, feature curves, and other conclusions lay the foundation for artificial intelligence system of mineragraphy recognizing and processing in sinter ore.
\end{abstract}

KEY WORDS: sinter ore mineragraphy; index of reflection; gray histogram; Gaussian distribution; feature extraction.

\section{Introduction}

The observation and measurement of mineragraphy of sinter ore are still by manual up to now, especially for mineragraphy recognition. As a result, the experience and skill of operator has great effect on accuracy of experiment report, and the reports maybe hardly credible once the raw stuff or processing parameters have changed a lot. Furthermore, it's a complex and of high labor intensity procedure that the sintering ore sample was got, processed, observed and measured under mineral microscope, so an excellent operator should be educated through long term practice and learning, wherever in university or company.

Digital image processing was created in 1920s. It has been developed quickly and used widely, after the thirdgeneration computer was invented. Image processing science and technique must infiltrate into other fields, and be used by them. ${ }^{1)}$ Image recognition technique, as the hot topic in Image Processing field, has got great achievement in many academic fields and production applications. For example, Image Recognition System for human face, fingerprint and illness are playing an important role in different fields. As for applications of minerals industry, many achievements have been got in different procedures. ${ }^{2-5)}$ So if we can take good advantage of Image Processing Technique to achieve auto mineragraphy recognition, it will greatly improve present experiment level. Accuracy and sufficient feature extraction is the primary requirement for a better intelligent image recognition system, so it's very senseful to research the relationship of mineragraphy feature and statistical data of digital image systematically, and get the digital expression method of image feature exactly.

The paper researched the mineragraphy feature of sinter ore by method of analyzing its gray histogram, and the deeper information saved in gray histogram was explored finally, so a good foundation was established for the intelligent mineragraphy recognition system.

\section{Mineragraphy Experiment}

Generally speaking, the manual observation and measurement procedure in mineragraphy for sinter ore can be described as follows:

Firstly, put the polished section of sintering ore sample under the mineral microscope, and adjust the parameters of device well until the image of mineragraphy can be seen clearly. Secondly, do the observation through ocular glass, and make sure of the mineral compositions. Lastly, calculate the minerals' content with the eye-measurement method or area method. ${ }^{6,7)}$ Change the measurement area by moving the sample and continue to do the observation and measurement same as before. For one sintering ore polished section, about more than ten areas are necessary to get a more accurate result.

The criterion of classifying different mineragraphy is important for the experiment. There are two key evidences of the criterion. One is the reflection of minerals, and the other is the texture feature about minerals under microscope. 
Table 1. Index of reflection of common minerals in sinter ore.

\begin{tabular}{cccccc}
\hline mineral & magnetite & hematite & fayalite & $\begin{array}{c}\text { calcium } \\
\text { silicate }\end{array}$ & $\begin{array}{c}\text { calcium } \\
\text { ferrite }\end{array}$ \\
\hline index of reflection & 20 & $22 \sim 25$ & $10 \sim 12$ & $5 \sim 8$ & $18 \sim 18.5$ \\
\hline
\end{tabular}

In practice, the texture feature of sinter ore is very difficult to describe and judge, so the reflection index of sinter ores become a major reference for classifying them most of the time. It describes how much luminous beam the mineral can reflect, so the index of reflection is defined as Eq. (1).

$$
R=\frac{I_{\mathrm{r}}}{I_{\mathrm{i}}}
$$

Where, $I_{\mathrm{r}}$ represents the intensity of reflect light, $I_{\mathrm{i}}$ the intensity of incident light, and the index of reflection $R$. Because $I_{\mathrm{r}}$ is always less than $I_{\mathrm{i}}, R$ expressed with \% customarily, is always less than one. The common minerals' index of refection in sinter ores are shown as Table $\mathbf{1 .}$

\section{Gray Histogram}

Gray histogram, which is an important feature of image, can be regarded as the approximate expression of a density function of gray. It shows the frequency of a fix gray appearing in an image. It is described as Eq. (2).

$$
G_{i}=\sum_{m=1}^{M} \sum_{n=1}^{N} P(i, m, n)
$$

Here, the $M$ and $N$ represent the total number of row and column, and the $G_{i}$ represents the appearance times of gray $i$, and the definition of $P(i, m, n)$ is described as Eq. (3).

$$
P(i, m, n)= \begin{cases}1 & P(m, n)=i \\ 0 & P(m, n) \neq i\end{cases}
$$

Where, $P(m, n)$ is the gray value of point $(m, n)$. As the sizes of images may be different in processing, there is nonsense to compare $G_{i}$ of different images. So Normalization Treating shown as Eq. (4) is necessary to Eq. (2).

$$
G_{i}^{\prime}=\frac{G_{i}}{T}
$$

Where, the $G_{i}{ }^{\prime}$ is an normalization value, and the $T$ represents the total amount of pixel of an image. Taking a micrograph of sinter ore like Fig. 1 as an example, Fig. $\mathbf{2}$ is its normalization gray histogram calculated by Eqs. (2), (3), and (4).

Generally speaking, the statistical variables as average and variance of gray in an image, as well as the maximum and total deviation of gray histogram of an image, can be used as the texture feature of a micrograph. But the conclusion that these statistical data do not have direct and clear relationship with the information, which, like mineral's category and mineral's content, we want to know most, can be got after carefully analyzing their equations and physical meaning. Therefore, more information hided in the gray histogram should be found out.

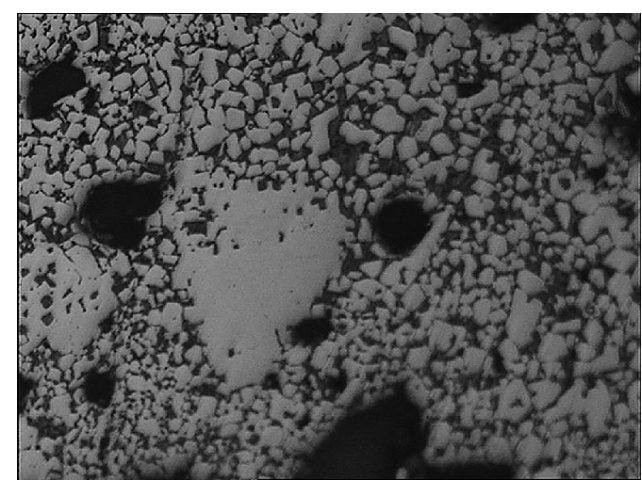

Fig. 1. Porphyritic structure of magnetite (reflected light $\times 500$ ).

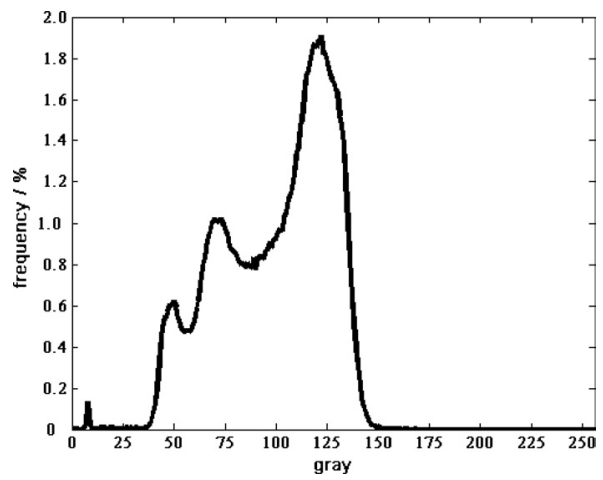

Fig. 2. Normalization gray histogram of porphyritic structure of magnetite.

In Fig. 2's curve, it can be found that there are three 'peaks', which represents high frequency of corresponding gray. While Fig. 1 also can be divided into three main zones as grayish-white zone, deep-gray zone, and black zone, according to brightness of image which is another expression of minerals' indexes of reflection. After the three zones were identified through other experiment methods, the conclusions were that grayish-white zone with granular structure was magnetite, deep-gray zone scattering around magnetite's zone was adhesive joint named fayalite, and the black zone represented cavity. Comparing Fig. 1's three 'peaks' with Fig. 2's three zones, is the conclusion right that 'peak' in gray histogram represents category and content of mineral? This proposition was researched through mathematical models on minerals' features in gray histogram in the following parts of the paper.

\section{Mathematical Models on Minerals' Features in Gray Histogram}

\subsection{Model of Peak-find in Gray Histogram}

To the curve known equation, the peaks and valley can be got by second differentiating. To the curve unknown equation, difference is used instead of differential. The method to judge peak or valley is described as Eq. (5).

$$
\Delta G_{i}^{\prime}=\left\{\begin{aligned}
1 & G_{i}^{\prime}-G_{i+1}{ }^{\prime}<-H \\
0 & \left|G_{i}{ }^{\prime}-G_{i+1}{ }^{\prime}\right|<H \\
-1 & G_{i}^{\prime}-G_{i+1}{ }^{\prime}>H
\end{aligned}\right.
$$

Where, $H$ refers to positive threshold which was set ac- 
Table 2. The relative features of normalization gray histogram.

\begin{tabular}{cccc}
\hline number of peaks & peak interval 1 & peak interval 2 & peak interval 3 \\
\hline 3 & $49 \sim 51$ & $68 \sim 73$ & $120 \sim 122$ \\
\hline
\end{tabular}

cording to specific image to reduce inaccuracy because of infinitesimal disturbance. $\Delta G_{i}{ }^{\prime}$ represents the tendency of curve at the point where gray equal to $i . \Delta G_{i}{ }^{\prime}=1$ means the curve ascends, $\Delta G_{i}{ }^{\prime}=-1$ means the curve falls down, and $\Delta G_{i}{ }^{\prime}=0$ means the curve is flat. Therefore, $1(0 \ldots 0)-1$ means the peak of curve, and $-1(0 \ldots 0) 1$ means the valley of curve. After the peak and valley were founded, regard the center of flat part as the position of peak or valley. The Fig. 2's peaks were extracted according to the method above, and the result is shown as Table 2.

\subsection{Calculated Model of Index of Reflection}

As the result of different index of reflection, the minerals have different brightness under the microscope's field of view, so the relationship between them can be built as Eq. (6).

$$
R=k \times \frac{I}{255}
$$

Where, $k$ represents the coefficient of correction of the model. The equation is based on two assumptions. One is that the black image with gray value of 0 is obtained when the index of reflection of mineral is 0 , and the other is that the white image with gray value of 255 is obtained when the index of reflection of mineral is 1 .

The reflection indexes of two minerals are assumed as Eq. (7) and Eq. (8) individually according to Eq. (6), so Eq. (9) can be got by dividing Eq. (7) with Eq. (8).

$$
\begin{gathered}
R_{1}=k \times \frac{I_{1}}{255} \\
R_{2}=k \times \frac{I_{2}}{255} \\
\frac{R_{1}}{R_{2}}=\frac{I_{1}}{I_{2}}
\end{gathered}
$$

Therefore, the ratios of reflection index of any two minerals was got as Table 3 according to Eq. (9). Data in Table 3 is the result that the reflection index of mineral in column is divided by the reflection index of mineral in row. So the data in diagonal line of table are all 1.00, and the product of two data in symmetry position of diagonal line is 1.00 . The gray of peak 3 was divided by the gray of peak 2 in Table 2, and then 1.71 was got which is right in the zone of 1.67-2.00 in Table 3, which is the ratio of magnetite and fayalite. Otherwise, the cavity in Fig. 1 is corresponding part of peak 1 in Fig. 2.

The features of data distribution in Table 3 were considered next. The first column of Table 3, with which the floating column chart as Fig. 3(A) was drawn, are about the ratios of reflection index of magnetite and other minerals. It's
Table 3. Ratio of reflection index of minerals (Col./Row).

\begin{tabular}{cccccc}
\hline ratio & magnetite & hematite & fayalite & $\begin{array}{c}\text { calcium } \\
\text { silicate }\end{array}$ & $\begin{array}{c}\text { calcium } \\
\text { ferrite }\end{array}$ \\
\hline magnetite & 1.00 & $1.10 \sim 1.25$ & $0.50 \sim 0.60$ & $0.25 \sim 0.40$ & $0.90 \sim 0.93$ \\
hematite & $0.80 \sim 0.91$ & 1.00 & $0.40 \sim 0.54$ & $0.20 \sim 0.36$ & $0.72 \sim 0.84$ \\
fayalite & $1.67 \sim 2.00$ & $1.83 \sim 2.08$ & 1.00 & $0.45 \sim 1.00$ & $1.50 \sim 1.85$ \\
calcium silicate & $2.50 \sim 4.00$ & $2.75 \sim 5.00$ & $1.00 \sim 2.20$ & 1.00 & $2.25 \sim 3.70$ \\
calcium ferrite & $1.08 \sim 1.11$ & $1.19 \sim 1.39$ & $0.54 \sim 0.66$ & $0.27 \sim 0.44$ & 1.00 \\
\hline
\end{tabular}

apparent that the columns in Fig. 3(A) are located in interval without any superposition in vertical axis. In Fig. 3(B), the columns are located in interval in vertical axis except a little superposition of magnetite and calcium ferrite. In Fig. $3(\mathrm{E})$, the columns are located in interval in vertical without any superposition. In Figs. 3(C) and 3(D), the columns are located with superposition in vertical, because the index of reflection of calcium silicate and fayalite are not fixed value, but vary in a relatively bigger range. When they are used as divider, the quotients' ranges are so bigger that the bars overlap a lot in vertical axis.

Whether the columns overlap in vertical axis or not mean whether the minerals are recognized according to their peaks' relatively positions in gray histogram. If they do not overlap, the mineral can be recognized correctly by its peak's position, while if they overlap a lot, it may cause mistake to judge the mineral only by its peak's position.

\subsection{Gaussian Gray Distribution Model of Mineral in Histogram}

The category and content of minerals influence the shape of curve of gray histogram directly. Generally speaking, if the content of a mineral in a mineragarphy image is low, or even none, the valley should appear in a certain position in its gray histogram, or else the peak should appear when a mineral is more in content. However, the third case maybe appear that the peak disappear or appear ambiguous when the content of several minerals satisfy a certain relationship. Taking Fig. 4 as an example, it's a classical corrosion structure of magnetite with calcium ferrite as the adhesive joint. The magnetite in this structure is residual anhedron with small grain size and approximately circular shape because of corrosion action, which mainly scatter round calcium ferrite. Due to the index of reflection of these two minerals are adjacent and their content agree with a certain relationship, there is only three peaks in its gray histogram as Fig. 5 shown. Peak 1 in 39-41 is the zone of cavity, the unapparent peak 2 in 74-76 because of effect of dense element points is the zone of adhesive joint of fayalite, and peak 3 in 110-112 is the zone of mixture zone of magnetite and calcium ferrite (The peak in $5-10$ is caused by the image capturing device after research on all the images and their gray histograms). It should be emphasized that the peak 3 and its adjacent zone represent two minerals. So the question, what's relationship the minerals' index of reflection and their content should agree with when the number of peaks in gray histogram represents the number of species of minerals, is coming if we want to regard the peaks of gray histogram as a very important feature in image recognition system. The conclusion will be given out after deducing in mathematics as follows. 

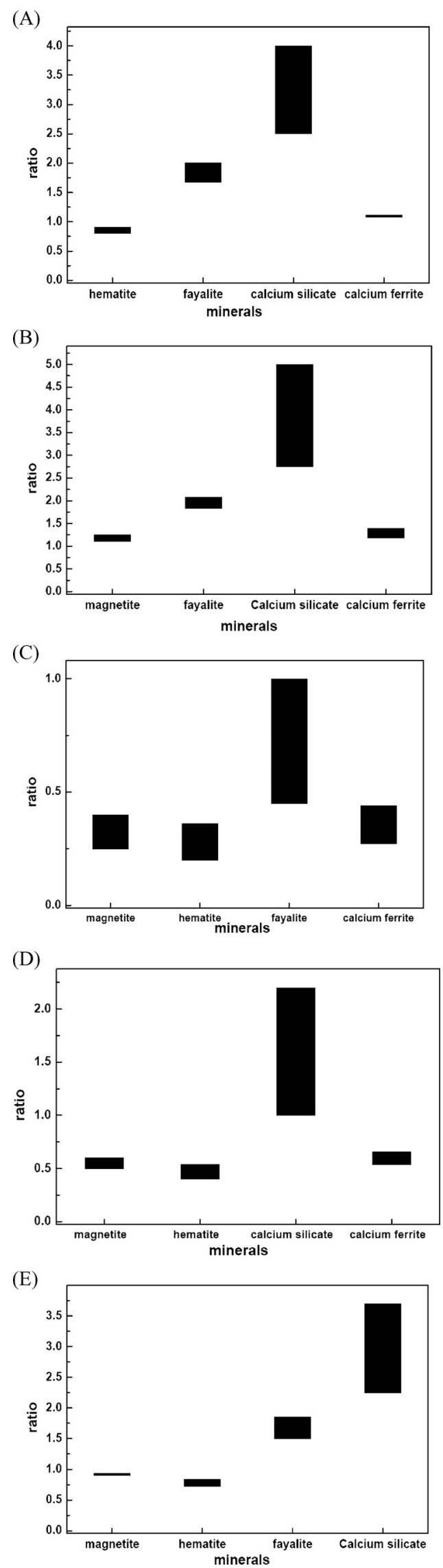

Fig. 3. Floating column charts of ratio of reflection of different minerals. (A) Magnetite, (B) hematite, (C) calcium silicate, (D) fayalite, (E) calcium ferrite.

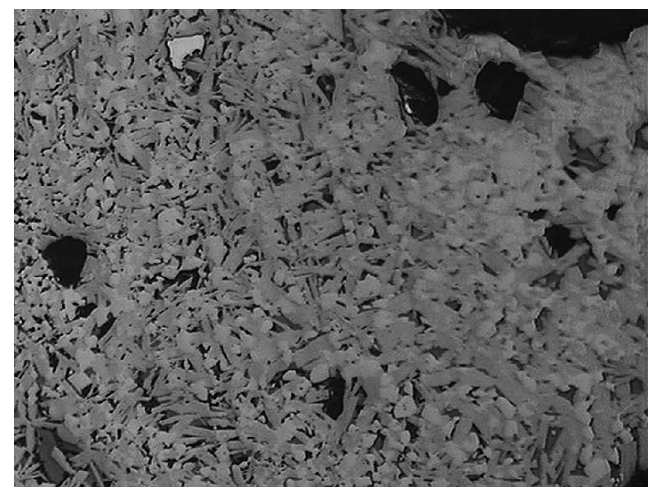

Fig. 4. Corrosion structure of magnetite and calcium ferrite (reflected light $\times 500$ ).

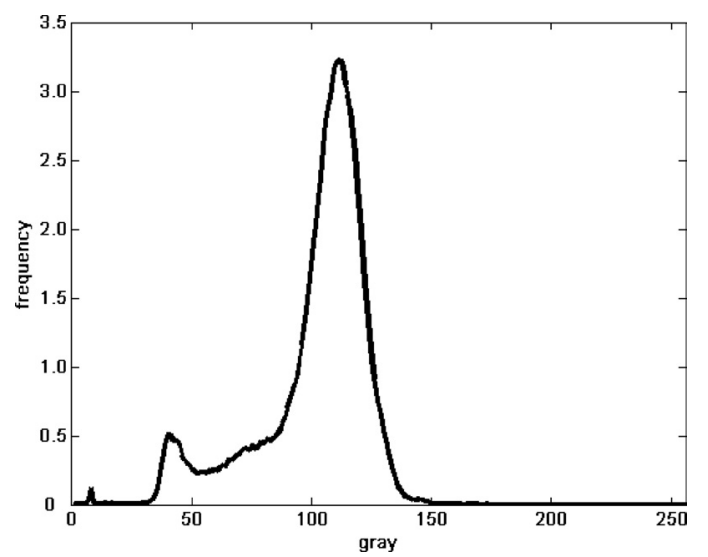

Fig. 5. Normalization gray histogram.

\subsubsection{Regularities of Gray Distribution of Single Mineral}

Theoretically speaking, in the gray histogram, minerals have definitive gray value which can be calculated by Eq. (6), however, in practice, the gray value of a mineral vary in a definitive zone. There are two reasons of this phenomenon: firstly, as a result of different crystallization status, minerals' indexes of reflection vary in ranges; secondly, the noise creates when the image signal is captured, saved, transferred, and A/D conversed. So the model of Gaussian distribution described as Eq. (10) was assumed to agree with the gray distribution of a mineral in gray histogram. ${ }^{8,9)}$

$$
I \sim N\left(\mu, \theta^{2}\right)
$$

Here, $I$ is gray distribution of mineral; $\mu$ and $\theta^{2}$, which represent mean value and variance of Gaussian distribution, can be calculated by Eqs. (11), (12) and (13) individually.

$$
\begin{gathered}
\mu=E(I)=\sum_{i=I_{\min }}^{I_{\max }} G_{i} \times \varphi(i) \\
E\left(I^{2}\right)=\sum_{i=I_{\text {min }}}^{I_{\max }} G_{i}^{2} \times \varphi(i) \\
\theta^{2}=D(I)=(E(I))^{2}-E\left(I^{2}\right) .
\end{gathered}
$$

Here, $I_{\max }$ and $I_{\min }$ represent the maximum and minimum in range of gray of mineral, $\left(I_{\max }\right.$ and $I_{\min }$ can be calculated 
Table 4. Regularities of gray distribution of minerals.

\begin{tabular}{cccccc}
\hline mineral & magnetite & hematite & fayalite & calcium silicate & calcium ferrite \\
\hline$\mu$ & 121.1 & 137.8 & 70.6 & 52.6 & 109.5 \\
$\theta^{2}$ & 34.0 & 19.6 & 67.9 & 47.5 & 128.7 \\
\hline
\end{tabular}

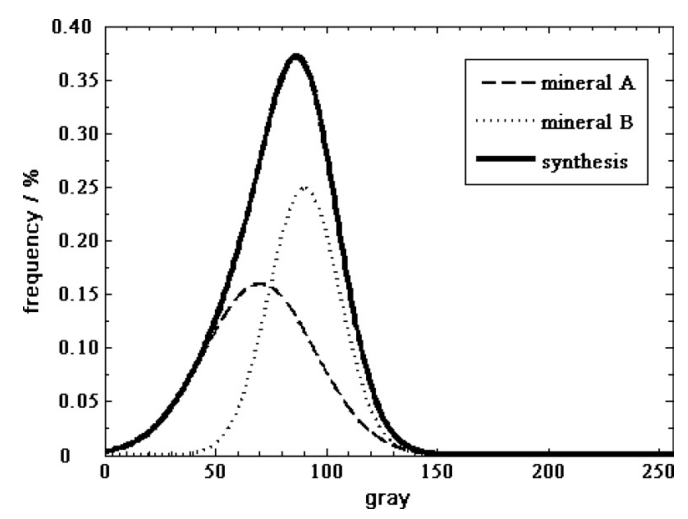

Fig. 6. Single peak of synthetical curve.

by Eq. (6), once the $k, R_{\max }$ and $R_{\min }$ are defined by experiment. Here, $R_{\max }$ and $R_{\min }$ represent the maximum and minimum in range of index of reflection of mineral; $k$ represents the amendment coefficient same with Eq. (7) and Eq. (8)). $\varphi(i)$ represents the frequency of gray $i$ appearing in certain zone. $\theta^{2}$, which is assumed to be constant to a mineral, can be calculated by averaging the experimental samples. So the regularities of gray distribution of minerals shown as Table 4 were got by Eqs. (11), (12) and (13).

\subsubsection{Regularity of Gray Distribution of Two Minerals}

The zone of gray distribution of different minerals may be in superposition, the synthetical curve of gray distribution is got from adding the two curves. Taking Fig. 6 as an example, mineral A and mineral $\mathrm{B}$ have superposition zone of gray distribution, as a result of synthesizing, only a peak appear in the gray histogram, however, two peaks appear in another condition as Fig. 7 shown.

Two minerals' gray distribution are regard as Eq. (14) and Eq. (15) according to assumption as Eq. (10), therefore, their individual density functions as Eq. (16) and Eq. (17) are gotten.

$$
\begin{array}{r}
G_{1} \sim N_{1}\left(\mu_{1}, \theta_{1}^{2}\right) \ldots \ldots \ldots \ldots . . . \\
G_{2} \sim N_{2}\left(\mu_{2}, \theta_{2}^{2}\right) \ldots \ldots \ldots \ldots \ldots \\
f_{1}(x)=\frac{C_{1}}{\sigma_{1} \sqrt{2 \pi}} e^{\frac{-\left(x-\mu_{1}\right)^{2}}{2 \sigma_{1}^{2}}} \ldots \ldots \\
f_{2}(x)=\frac{C_{2}}{\sigma_{2} \sqrt{2 \pi}} e^{\frac{-\left(x-\mu_{2}\right)^{2}}{2 \sigma_{2}^{2}}} \ldots \\
f(x)=\sum_{i=1}^{N} \frac{C_{i}}{\sigma_{i} \sqrt{2 \pi}} e^{\frac{-\left(x-\mu_{i}\right)^{2}}{2 \sigma_{i}^{2}}}
\end{array}
$$

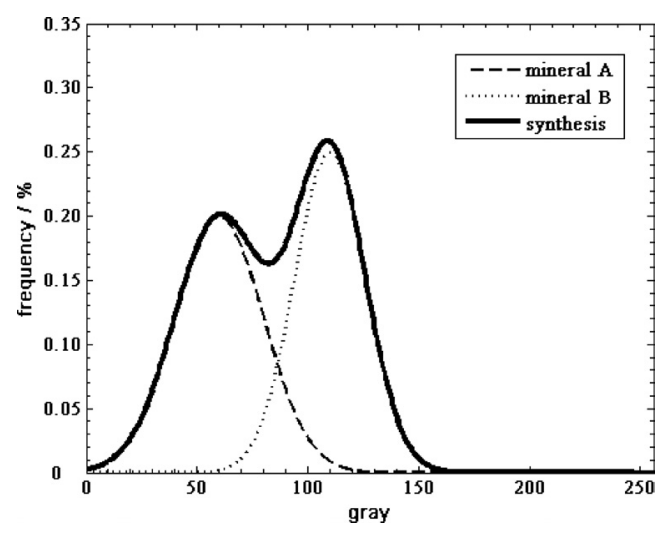

Fig. 7. Two peaks of synthetical curve.

$$
\begin{aligned}
\frac{d\left(f_{1}(x)+f_{2}(x)\right)}{d x}= & \frac{C_{1}\left(x-\mu_{1}\right) \theta_{2}^{3}}{\sqrt{2 \pi}} e^{\frac{-\left(x-\mu_{1}\right)^{2}}{2 \sigma_{1}^{2}}} \\
& +\frac{C_{2}\left(x-\mu_{2}\right) \theta_{1}^{3}}{\sqrt{2 \pi}} e^{\frac{-\left(x-\mu_{2}\right)^{2}}{2 \sigma_{2}^{2}}}=0
\end{aligned}
$$

Where, $C_{1}$ and $C_{2}$ represent the mineral contents. The synthetical function, just like Eq. (18) with $i$ equal to 2, was got by adding Eq. (16) and Eq. (17). The peak distribution in gray histogram is a kind of graph expression of extreme points of synthetical function. So the extreme points distribution can be analyzed by differentiating synthetical function as Eq. (19) shown. The point that makes Eq. (18) equal to zero is the position of peak or valley (flat ground) appearing in gray histogram. When $x=\left[\mu_{1}, \mu_{2}\right]$, if there are three roots which make Eq. (19) equal to zero, it indicates that there are two peaks and one valley in $\left[\mu_{1}, \mu_{2}\right]$ of gray histogram, because $\mu_{1}$ and $\mu_{2}$ are absolutely not the position of valley appearing in gray histogram. If there are two roots of Eq. (19) in $\left[\mu_{1}, \mu_{2}\right]$, it indicates that there are one peak and one valley in $\left[\mu_{1}, \mu_{2}\right]$ of gray histogram. If there are only one root of Eq. (19), it means that only one peak appearing in gray histogram without any valley.

To one's disappointed, the Eq. (18) is a transcendental equation which is too difficult to get a analytic solution. However, the regularity of solution distribution can be got by graphic mean and numerical method. The features of curve of two minerals in gray histogram were got by using the method above.

The solution of Eq. (19) in condition of magnetite and hematite were studied by changing the mineral content by 10 percent every step, the result was shown as Table 5. Figure 8 gives the theoretical gray histogram of magnetite and hematite in four special conditions according to their feature of gray distribution and the model of Gaussian distribution. Figure 9 show the solution of Eq. (19) in different ratio of magnetite and hematite.

The followings can be concluded from the Fig. 8 and 
Table 5. The solution of Eq. (18) in different minerals' content.

\begin{tabular}{ccccc}
\hline & $\mu_{1}=121.1$ & $\mu_{2}=137.8$ & $\sigma_{1}=5.83$ & $\sigma_{2}=4.42$ \\
\\
\hline $\mathrm{C} 1 \%$ & $\mathrm{C} 2 \%$ & Max. root & Mid. root & Min. root \\
10 & 90 & 136.98 & 122.91 & 121.97 \\
20 & 80 & 136.95 & 125.33 & 121.25 \\
30 & 70 & 136.92 & 126.62 & 121.13 \\
40 & 60 & 136.88 & 127.67 & 121.08 \\
50 & 50 & 136.82 & 128.66 & 121.05 \\
60 & 40 & 136.72 & 129.70 & 121.03 \\
70 & 30 & 136.54 & 130.93 & 121.02 \\
80 & 20 & 136.03 & 132.76 & 121.01 \\
90 & 10 & 135.70 & 135.70 & 121.00 \\
\hline
\end{tabular}

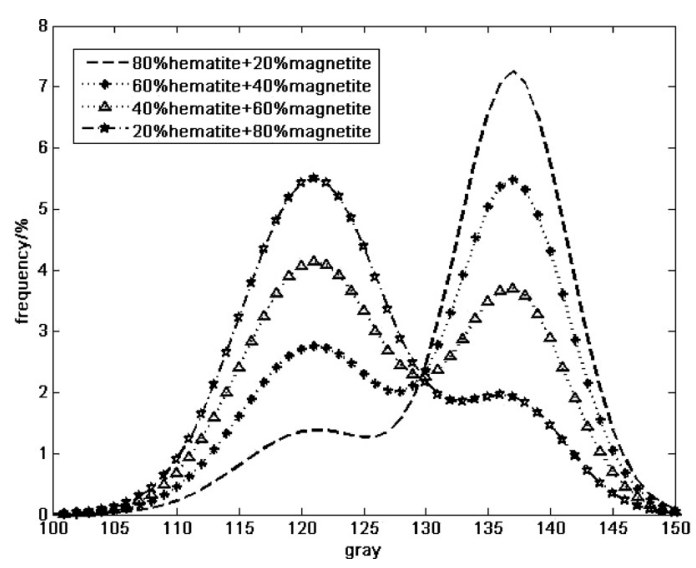

Fig. 8. Synthetical gray histogram of hematite and magnetite in different ratios.

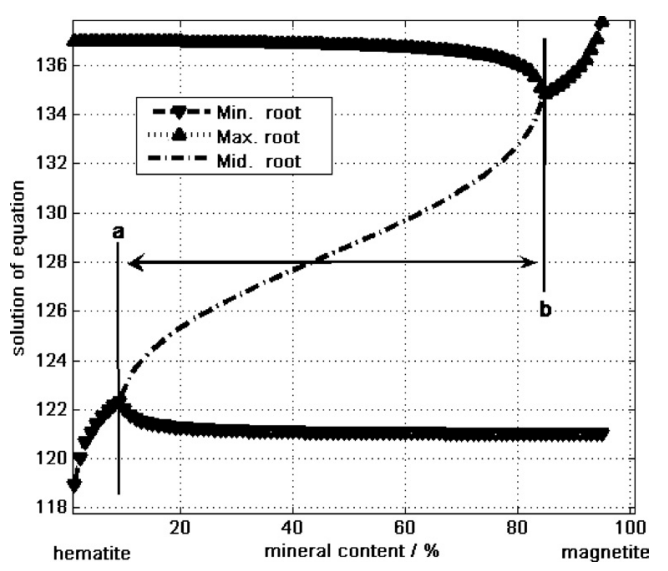

Fig. 9. Solution of Eq. (19) of hematite and magnetite in different ratios.

Fig. 9.

1. A mineral's peak becomes sharper and more obvious, and the position of peak becomes more closed to the theoretical position which is the mean value of gray distribution, with content of mineral increasing.

2. When the ratio of the two mineral is beyond a critical value, there are three roots of Eq. (19) in $\left[\mu_{1}, \mu_{2}\right]$, so two peaks and a valley appear. Taking Fig. 9 as an example, line $a$ and line $b$ is the critical position of changing from two peaks to single peak in the gray histogram. When the ratio of two minerals varies in the range of line a to line $b$, there are three roots of Eq.
(19), so two peaks and a valley appear in Fig. 8. Beyond the range of line $a$ and line $b$, only two roots of Eq. (19), which represent a peak and a valley (flat ground) in the gray histogram.

The same methods above were used to analyze other minerals' combination, and the results show as Fig. 10.

\subsection{Modeling Verification}

Taking Fig. 11 as an example to verify the models above and Fig. 12 is its normalization gray histogram. The minerals' contents were measured by other method like eyemeasurement method or area method mentioned above, so the result $34 \%$ hematite, $8 \%$ magnetite and $58 \%$ calcium ferrite were got. The theoretical gray histogram as Fig. 13 was drew according the model like Eq. (18).

Comparing Fig. 12 with Fig. 13, it can be found that the curves of two figures are similar in the zone between 75 and 150. The great difference of zone with gray value less than 75 was caused because of the cavity in Fig. 11, and the model in this paper hasn't considered the influence of cavity because of its more complex regularity of gray distribution. Considering the reason of cavity, the two figure's similarity verifies the rationality of Gaussian gray distribution model of mineral in gray histogram.

Table 6 shows the positions of peaks. 0.81 , the ratio of peak 2 and peak 3, just in range of $0.80-0.91$, which is the ratio of magnetite and hematite in Table 3, also in range of $0.72-0.84$, which is the ratio of calcium ferrite and hematite in Table 3. Therefore, it indicates the rationality of model of peak find and model of index of reflection.

The example above shows that the gray histogram of sinter ore's mineragraphy image represents the information about mineral's category and content which can be calculated out by the models developed in this paper. The research on how to get the category and content with Genetic Algorithm to realize the best fitting will be done next step.

\section{Conclusions}

The conclusion as followings were got after analyzing the gray histogram with the model of peak-find, calculated model of index of reflection, and Gaussian gray distribution model of mineral in histogram in this paper.

(1) The relatively position of peak in the gray histogram represents special relationship of category and composition of minerals, which were got by using the model of index of reflection in theory.

(2) The parameters like $\mu$ and $\theta^{2}$ of common minerals were got by statistical averaging with Gaussian gray distribution model. With these two parameters and Gaussian gray distribution model, the curve of gray histogram can be fitted in a certain category and content.

(3) The feature curve of gray histogram of two minerals' was got by synthesizing two density functions.

(4) The feature index like number and position of peak and valley of two minerals in different ratio were got by differentiating the distribution functions.

(5) The feature index, feature curve, and other conclusions make the foundation for building artificial intelligence system of mineragraphy recognizing and processing of sintering ore. 
ISIJ International, Vol. 48 (2008), No. 2

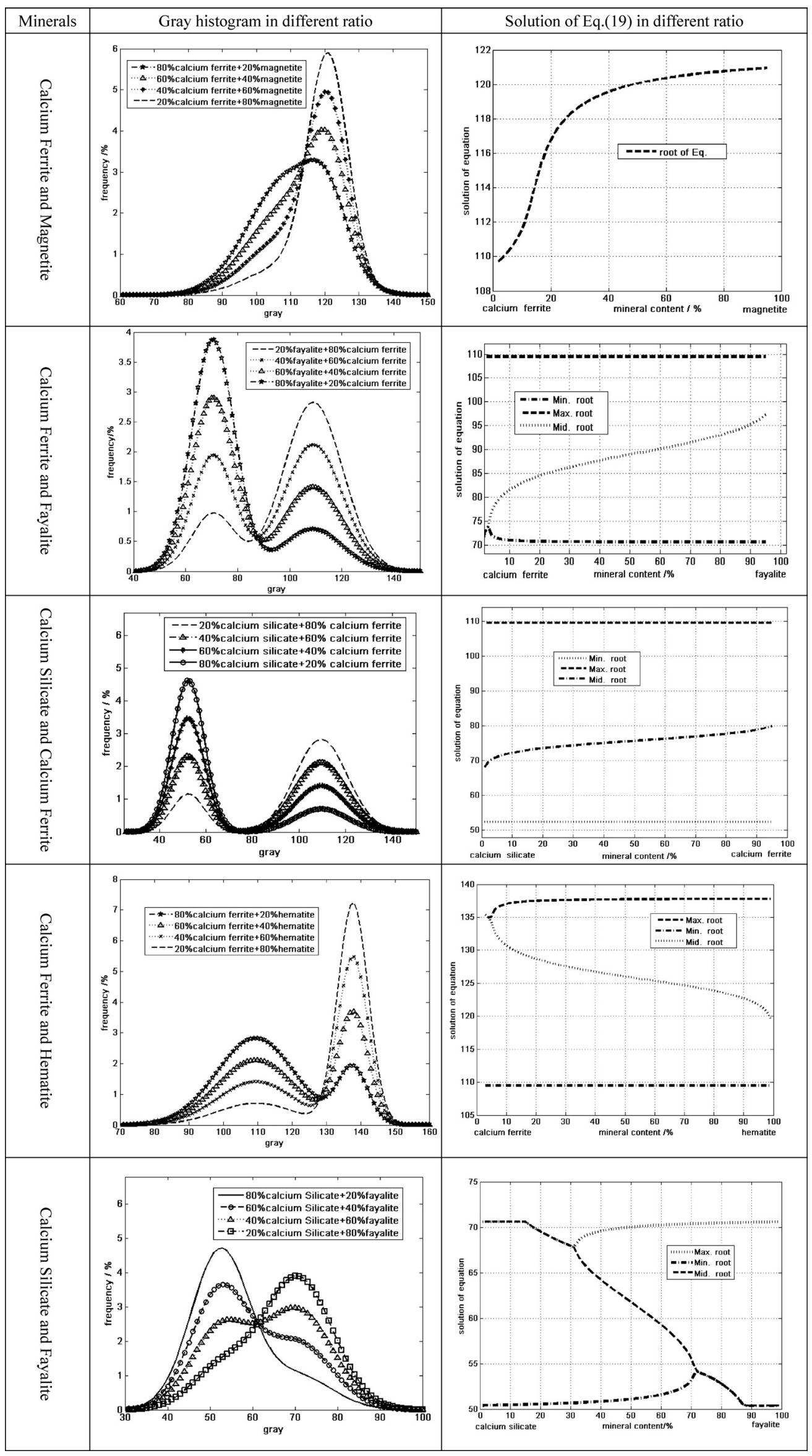

Fig. 10. Synthetical gray histogram and solution of Eq. (19) of different minerals. 


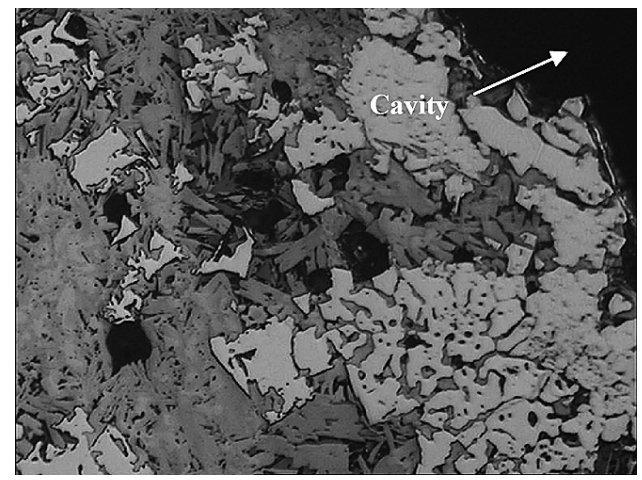

Fig. 11. Microstructure of magnetite and hematite (reflected light $\times 500)$.

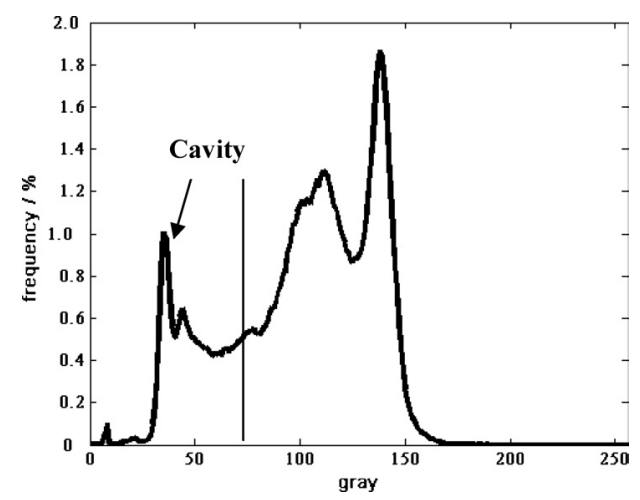

Fig. 12. Normalization gray histogram.

Table 6. The relative features of normalization gray histogram.

\begin{tabular}{cccc}
\hline peaks & peak Interval 1 & peak Interval 2 & peak Interval 3 \\
\hline gray value & $34 \sim 35$ & $110 \sim 112$ & $137 \sim 138$ \\
ratio & 0.26 & 0.81 & 1 \\
\hline
\end{tabular}

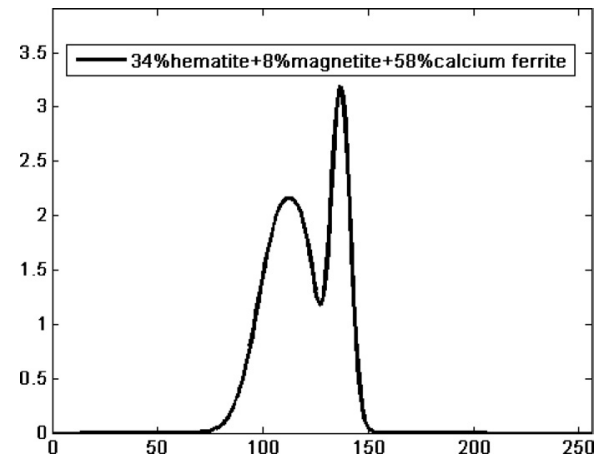

Fig. 13. The gray histogram in theory.

\section{Acknowledgement}

The authors are especially grateful to Chongqing University Postgraduates' Science and Innovation Fund (200701Y1B0020188) and Science Foundation of Ministry of Education of China.

\section{REFERENCES}

1) R. C. Gonzalez: Digital Image Processing, Publishing House of Electronics Industry, Beijing, (1981), 6.

2) R. Marschallinger: Comput. Geosci., 23 (1997), 119.

3) J. V. Smith and E. Beermann: Comput. Geosci., 33 (2007), 335.

4) C. V. Alphen: Miner. Eng., 20 (2007), 496.

5) M. Teresa, A. Andres, Q. Xavier, F. Oriol and G. Wes: Int. J. Coal Geol., 71 (2007), 122.

6) Y. Ren: Facieology and Mineragraphy in Iron \& Steel Metallurgy, Metallurgical Industry Press, Beijing, (1982), 105.

7) L. Zhou: Technologic Mineralogy, Metallurgical Industry Press, Beijing, (1982), 105.

8) J. Xue, Y. Zhang and X. Lin: Acta Electr. Sin., 27 (1999), 95.

9) J. Chang, K. Fan and Y. Chang: Image Vis. Comput., 20 (2002), 203. 